\title{
Predictive Value of the Standardized Uptake Value of 18F-fluorodeoxyglucose Positron Emission Tomography-Computed Tomography in Assessing Axillary Lymph Node Metastasis in Different Subtypes of Breast Cancer
}

\author{
Nawon Kim, M.D. ${ }^{1 *}$, Jihye Choi, M.D. ${ }^{1,2 *}$, Eun-Byeol Ko, M.D. ${ }^{1}$, Sang Hee Kim, M.D. ${ }^{1}$, Jinwoo Jun, M.D. ${ }^{1}$, \\ Hyun-Ah Kim, M.D., Ph.D. ${ }^{1}$, Woo Chul Noh, M.D., Ph.D. ${ }^{1}$, Min-Ki Seong, M.D. ${ }^{1}$ \\ ${ }^{1}$ Department of Surgery, Korea Cancer Center Hospital, Korea Institute of Radiological and Medical Sciences, Seoul; ${ }^{2}$ Department of Surgery, National \\ Medical Center, Seoul, Korea
}

Purpose: This study aimed to examine the association between the maximum standardized uptake value (SUVmax) of different molecular subtypes of primary breast cancer with axillary lymph node (ALN) metastasis. Methods: The medical records of 633 patients, who underwent 18F-fluorodeoxyglucose positron emission tomography-computed tomography (PET/CT) for preoperative staging, were retrospectively reviewed. The cohort was stratified by the following molecular subtypes with immunohistochemical examination: luminal A, luminal B, human epidermal growth factor receptor 2 positive (HER2+), and triple negative. We evaluated the optimal cutoff SUVmax to predict ALN metastasis in each subtype using the receiver operating characteristic (ROC) analysis. Moreover, the risk factors for ALN metastasis were evaluated. Results: Overall, the SUVmax was positively correlated with the number of metastatic ALN $(p=0.001)$. The mean SUVmax was higher in aggressive subtypes $(4.5 \pm 0.2,6.1 \pm 0.4,6.5 \pm 0.5$, and $7.5 \pm 0.5$ in luminal $A$, luminal $B, H E R 2+$, and triple negative, respectively, $p<0.001)$. Upon ROC analysis, the SUVmax of the HER2+ subtype predicted ALN metastasis most accurately, with a cutoff value of 5.5 , area under the curve (AUC) of 0.708 , sensitivity of $74.2 \%$, and specificity of $64.6 \%(p=0.002)$. The triple negative subtype did not show a significant difference in SUVmax between patients with and without metastasis $(p=0.13)$. Subtype-adjusted SUVmax, HER2 positivity, lymphovascular invasion, and T stage were significant predictors for ALN metastasis. Conclusion: The SUVmax of primary breast cancer may be an independent predictor of ALN metastasis, being the most accurate in the HER2+ subtype. As PET/CT could facilitate tailored axillary management, this approach could be considered for the initial staging and treatment planning in patients with breast cancer.

Key Words: Breast neoplasms; Lymphatic metastasis; Positron emission tomography computed tomography; ROC curve

\section{INTRODUCTION}

Trends in the management of the axilla in patients with early breast cancer have rapidly evolved toward a conservative and less extensive treatment. For the last few decades, axillary lymph node (ALN) dissection had been recommended as the standard procedure in patients with defined ALN metastasis because it was the most accurate method

Correspondence: Min-Ki Seong, M.D.

Department of Surgery, Korea Cancer Center Hospital, Korea Institute of Radiological and Medical Sciences, 75 Nowon-ro, Nowon-gu, Seoul 01812, Korea Tel: +82-2-970-1251, Fax: +82-2-970-2419, E-mail: mklegend@kcch.re.kr

*These authors have contributed equally to this work.

This study was supported by a grant of the Korea Institute of Radiological and Medical Sciences (KIRAMS), funded by Ministry of Science and ICT (MSIT), Republic of Korea (No. 50472-2018).

Received: Aug 6, 2019 Revised: Sep 5, 2019 Accepted: Oct 23, 2019 of identification and an important adverse prognostic factor for these patients [1]. However, evidence from recent clinical trials, such as ACOSOG Z0011 and AMAROS, suggested a practice change. As complete ALN dissection did not improve survival in both trials on the basis of effective radiotherapy, de-escalation of axillary surgery is currently allowed in patients with early breast cancer [2,3]. Hence, preoperative prediction of ALN status is becoming increasingly important in the initial treatment planning. Moreover, due to the rapid advancement in the understanding of breast cancer's molecular biology, we now recognize breast cancer as a heterogeneous disease that needs an individualized treatment approach. Therefore, noninvasive methods providing information on the biologic activity of the tumor and lymph node status preoperatively may be helpful in these patients.

Positron emission tomography-computed tomography (PET/CT) is 
a widely used imaging tool for evaluating the tumor stage and detecting disease recurrence [4]. PET/CT has advantages over other imaging modalities, as it is capable of showing abnormal metabolic activities prior to the detection of morphological changes in anatomic images. Additionally, PET/CT is useful in predicting lymph node metastasis in patients with cancer [5]. However, in patients with breast cancer, the role of PET/CT in axillary staging has not yet been fully established and has not been recommended for routine examinations [6]. The predictive value of the maximum standardized uptake value (SUVmax) parameter in detecting ALN metastasis among different breast cancer molecular subtypes remains controversial $[7,8]$.

This study aimed to evaluate if the preoperative SUVmax of primary tumor could predict ALN metastasis in patients with different molecular subtypes of breast cancer.

\section{METHODS}

\section{Study design and patient selection}

A total of 633 patients who underwent 18F-fluorodeoxyglucose (FDG) PET/CT for the initial staging of primary breast cancer between January 2010 and June 2015 were retrospectively enrolled in this study. Patients with carcinoma in situ, de novo stage IV breast cancer, history of neoadjuvant chemotherapy, and diagnosis of N1mi disease or other primary malignancies were excluded. Staging was performed according to the American Joint Committee on Cancer 7 th edition guidelines [9]. Data including patient age $(<50$ or $\geq 50$ years), tumor size (T stage, T1-T4), nodal status (N stage, N0-N3), hormone receptor status, human epidermal growth factor receptor (HER)-2 status, histologic grade (Grades 1, 2, and 3), and lymphovascular invasion (LVI) were obtained from our database, a web-based system that has been collecting information on patients with breast cancer since 1983. The need for informed consent was waived by the Institutional Review Board of the Korean Cancer Center Hospital, which approved the study protocols, due to the retrospective nature of this study (No. 2019-07-012). All procedures performed in studies involving human participants were in accordance with the 1964 Declaration of Helsinki and its later amendments.

\section{Immunohistochemical staining}

Formalin-fixed, paraffin-embedded tissue sections were obtained from the surgical specimen and routine immunohistochemical assessment for estrogen receptor (ER), progesterone receptor (PR), HER2, and Ki67 expression was performed. ER and PR positivity were defined as the expression of the proteins in at least $1 \%$ of all tumor cells. HER2 overexpression was defined as a score of $3+$ in the immunohistochemical staining or $2+$ with positive gene amplification in in situ hybridization. The Scarff-Bloom-Richardson grading system was applied for tumor grading. According to the recommendation of the 12th St Gallen International Breast Cancer Consensus Conference (2011), breast cancers were classified into four molecular subtypes: luminal A (ER- and/or PR-positive, Ki-67<14\%, and HER2-negative), luminal B (ER- and/or PR-positive, Ki-67 > 14\%, and HER2-negative or ER and/or PR-positive, any Ki-67 index, and HER2-positive), HER2+ (ER negative, PR-negative, and HER2-positive), and triple negative (ER-negative, PR-negative, and HER2-negative) [10].

\section{FDG PET acquisition}

All patients fasted for at least 6 hours prior to 18 F-FDG administration $(7.4 \mathrm{MBq} / \mathrm{kg}$ of body weight, intravenously) and had blood glucose levels $<7.2 \mathrm{mmol} / \mathrm{L}$. PET/CT was performed using a Biograph 6 PET/CT scanner (Siemens Medical Solutions, Knoxville, USA). PET/ CT images from the vertex to the upper thigh (5-6 bed positions) were acquired 60 minutes after 18F-FDG injection. First, CT images without contrast enhancement were obtained using a 6-slice helical CT scanner (peak voltage of $130 \mathrm{kVp}$, automated tube current of 30 $\mathrm{mA}, 0.6$ second/CT rotation, and pitch of 6). Then, PET emission data were obtained in the same area for 3.5 minutes per bed position. PET images were reconstructed using a conventional iterative algorithm (ordered subsets expectation-maximization, 2 iterations, and 8 subsets), using CT data for attenuation correction.

\section{Imaging analysis}

All PET/CT images were reviewed on an e.soft workstation (Siemens Medical Systems, Iselin, USA). For each patient, a region of interest (ROI) was manually placed on the entire breast lesion using a volumetric analysis template. The SUVmax of the lesion was calculated using decay-corrected activity within the ROI, injected dose, and body weight. All PET/CT images were reviewed and interpreted by three experienced nuclear medicine physicians, and decisions were made based on consensus. 


\section{Statistical analysis}

We compared discrete variables using the chi-square and Fisher's exact tests. Student's t-test on independent variables and analysis of variance were used to compare continuous variables, including mean age and SUVmax between different groups. Results are presented as frequencies and percentages for categorical variables or means \pm standard deviation for continuous variables. Receiver operating characteristic (ROC) analysis was performed to determine the ideal cutoff SUVmax for detecting ALN metastasis. Univariate and multivariate analyses on the predictors of ALN metastasis detection were performed using logistic regression. Statistical analyses were performed using the SPSS version 25 software (SPSS, Chicago, USA). A p-value $<0.05$ was considered statistically significant.

\section{RESULTS}

\section{Patient characteristics}

A total of 633 patients with operable, invasive breast cancer were included in the study. Overall, 370 patients (58.5\%) were node-positive, and $263(41.5 \%)$ were node-negative. The mean age was $51.8 \pm 0.4$ years. Tumor staging was as follows: stage I in 255 patients (40.3\%), stage II in 281 (44.3\%), and stage III in 97 (15.3\%). The molecular cancer subtypes were as follows: luminal A in 366 patients (57.8\%), luminal B in 57 (9.0\%), HER2 positive in 114 (18.1\%), and triple negative in 95 (15.1\%). The mean SUVmax of the cohort was $5.4 \pm 0.2$ (Table 1). Clinicopathological findings according to each molecular subtype are presented in Table 2. The percentage of patients with ALN metastasis differed among molecular subtypes $(p<0.001)$.

\section{Association of SUVmax and ALN metastasis}

In the entire cohort, the mean SUVmax of patients with and without ALN metastasis were $6.3 \pm 3.9$ and $4.8 \pm 3.9$, respectively (Table 3, $p<0.001)$. There was a positive correlation between the increase in SUVmax and number of metastatic ALNs $(p<0.001)$.

When stratified by subtypes, we found a significant positive correlation between the SUVmax and more biologically aggressive tumors: HER2+ and triple negative subtypes presented higher SUVmax than luminal A and luminal B subtypes (Figure 1). The mean SUV$\max$ were $4.5 \pm 0.2,6.1 \pm 0.4,6.5 \pm 0.5$, and $7.5 \pm 0.5$ in luminal A, luminal B, HER2+ and triple negative subtypes, respectively (Table 4,
Table 1. Overall patient characteristics $(n=633)$

\begin{tabular}{|c|c|c|}
\hline Characteristic & & №. (\%) \\
\hline Age & Mean \pm SD (range) & $51.8 \pm 0.4(15-91)$ \\
\hline \multirow[t]{4}{*}{ T stage } & $\mathrm{T} 1$ & $346(54.7)$ \\
\hline & $\mathrm{T} 2$ & $274(43.3)$ \\
\hline & $\mathrm{T} 3$ & $11(1.7)$ \\
\hline & $\mathrm{T} 4$ & $2(0.3)$ \\
\hline \multirow[t]{4}{*}{ N stage } & No & $370(58.5)$ \\
\hline & $\mathrm{N} 1$ & $169(26.7)$ \\
\hline & N2 & $51(8.0)$ \\
\hline & N3 & $43(6.8)$ \\
\hline \multirow[t]{3}{*}{ Stage (AJCC7th) } & 1 & $255(40.3)$ \\
\hline & $\|$ & $281(44.3)$ \\
\hline & III & $97(15.3)$ \\
\hline \multirow[t]{2}{*}{ ALN metastasis } & Positive & $370(58.5)$ \\
\hline & Negative & $263(41.5)$ \\
\hline \multirow[t]{3}{*}{ Histologic grade } & $1 / 2$ & $352(55.6)$ \\
\hline & 3 & $252(39.8)$ \\
\hline & Unknown & $29(4.6)$ \\
\hline \multirow[t]{2}{*}{ ER } & Positive & $429(67.8)$ \\
\hline & Negative & $204(32.2)$ \\
\hline \multirow[t]{2}{*}{ HER2 } & Positive & $172(27.1)$ \\
\hline & Negative & $461(72.7)$ \\
\hline \multirow[t]{3}{*}{ Ki67 } & High & $215(33.9)$ \\
\hline & Low & $281(44.3)$ \\
\hline & Unknown & $137(21.8)$ \\
\hline \multirow[t]{3}{*}{ LVI } & Positive & $143(22.6)$ \\
\hline & Negative & $487(76.9)$ \\
\hline & Unknown & $3(0.5)$ \\
\hline \multirow[t]{4}{*}{ Subtypes } & Luminal A & $366(57.8)$ \\
\hline & Luminal B & $57(9.0)$ \\
\hline & HER2+ & $114(18.1)$ \\
\hline & Triple negative & $95(15.1)$ \\
\hline \multirow[t]{2}{*}{ Operation Method } & BCS & $381(60.2)$ \\
\hline & Mastectomy & $252(39.8)$ \\
\hline \multirow[t]{4}{*}{ Types of axillary surgery } & SLNB & $377(59.6)$ \\
\hline & SLNB+ALND & $107(16.9)$ \\
\hline & ALND & $147(23.2)$ \\
\hline & No operation & $2(0.3)$ \\
\hline SUVmax & Mean \pm SD (range) & $5.4 \pm 0.2(0-33)$ \\
\hline
\end{tabular}

$E R=$ estrogen receptor; $\mathrm{PR}=$ progesterone receptor; $\mathrm{HER} 2$ = human epidermal growth factor receptor 2; $\mathrm{LVI}=$ lymphovascular invasion; $\mathrm{ALND}=$ axillary lymph node dissection; SLNB = sentinel lymph node biopsy; $B C S=$ breast conserving surgery; SUVmax = maximum standardized uptake value.

$p<0.001)$. Regarding ALN metastasis, it was associated with the highest frequency to the HER+ subtype (57\%), while the lowest frequency was associated with the triple negative subtype (28\%, Table 2). Luminal A, luminal B, and HER2+ subtypes showed a statistically significant difference between the primary tumor SUVmax and presence of ALN metastasis. Conversely, we found no significant differences in the triple negative subtype (Table $4, p=0.130$ ). 
Table 2. Clinico-pathological findings of each molecular subtype

\begin{tabular}{|c|c|c|c|c|c|}
\hline Characteristic & $\begin{array}{c}\text { Luminal } A(n=366) \\
\text { No. }(\%)\end{array}$ & $\begin{array}{c}\text { Luminal B }(n=57) \\
\text { No. }(\%)\end{array}$ & $\begin{array}{c}\text { HER2+ }(n=114) \\
\text { No. }(\%)\end{array}$ & $\begin{array}{c}\text { Triple negative }(n=95) \\
\text { No. }(\%)\end{array}$ & $p$-value \\
\hline Age $^{*}$ & $51.5 \pm 0.5$ & $50.7 \pm 1.2$ & $51.8 \pm 1.2$ & $53.8 \pm 1.2$ & 0.199 \\
\hline Tumor size (mm) & $19.7 \pm 0.6$ & $21.9 \pm 1.3$ & $22.5 \pm 1.8$ & $23.4 \pm 1.5$ & 0.036 \\
\hline \multicolumn{6}{|l|}{ Lymph node metastasis } \\
\hline Node negative patients & $216(59.0)$ & $36(63.2)$ & $49(43.0)$ & $68(71.6)$ & $<0.001$ \\
\hline Node positive patients & $150(41.0)$ & $21(36.8)$ & $65(57.0)$ & $27(28.4)$ & \\
\hline Numbers of metastatic lymph nodes* & $1.8 \pm 0.2$ & $2.4 \pm 0.4$ & $2.4 \pm 0.6$ & $1.3 \pm 0.4$ & 0.250 \\
\hline
\end{tabular}

HER2 = human epidermal growth factor receptor 2.

*Mean \pm SD.

Table 3. Correlation between the mean SUV max of primary tumor and degree of axillary lymph node metastasis

\begin{tabular}{lccccc}
\hline Node stage & N0 $(n=370)$ & N1 $(n=169)$ & N2 $(n=51)$ & N3 $(n=43)$ & $p$-value \\
\hline SUV max* & $4.7 \pm 3.9$ & $5.9 \pm 3.7$ & $7.2 \pm 4.7$ & $6.5 \pm 3.7$ & $<0.001$ \\
\hline
\end{tabular}

SUVmax $=$ maximum standardized uptake value.

*Mean \pm SD.

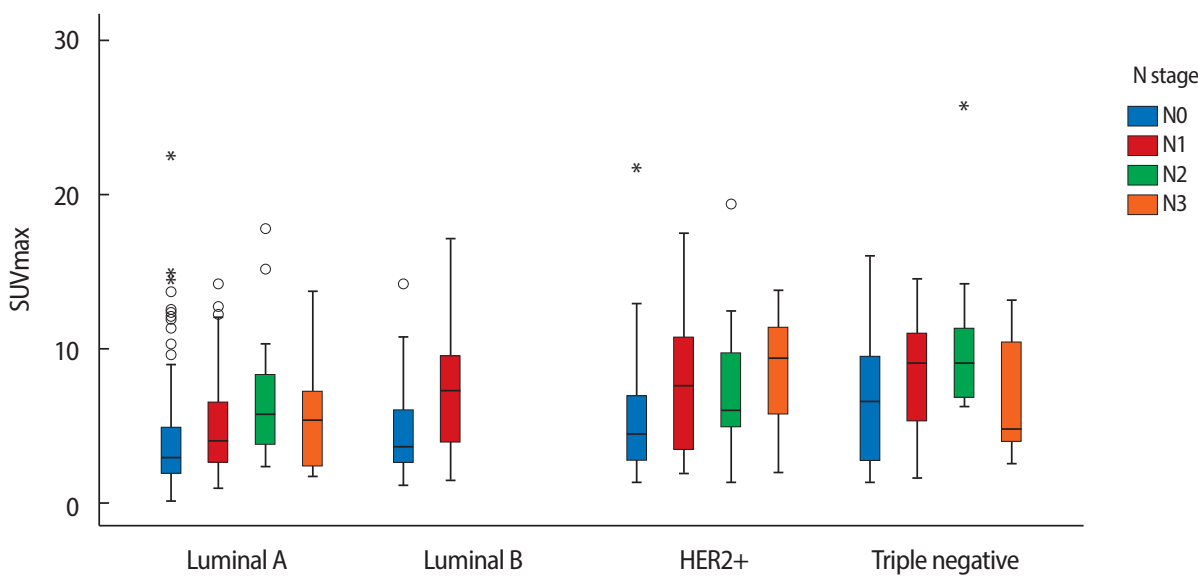

Figure 1. Correlation between the SUV max and axillary lymph node metastasis according to molecular subtype $(p=0.001)$.

\section{Cutoff SUVmax according to molecular subtypes}

We generated the ROC curves to determine the optimal cutoff SUVmax in the detection of ALN metastasis (Figure 2). In the overall population, the AUC was 0.641 (95\% confidence interval [CI], 0.5980.684; $p<0.001$ ), cutoff SUVmax was 3.6, sensitivity was 70.0\%, and specificity was $54.1 \%$. In the subtype analysis, the SUVmax in the HER2+ subtype predicted lymph node metastasis most accurately with an AUC of 0.708 , a cutoff SUVmax of 5.5, sensitivity of $74.2 \%$, and specificity of $64.6 \%(p=0.002)$. In the luminal A subtype, the AUC was 0.653 (95\% CI, 0.597-0.709; $p<0.001$ ), cutoff SUVmax was 3.5 , sensitivity was $64.7 \%$, and specificity was $58.8 \%$. In the luminal B subtype, the AUC was 0.684 (95\% CI, 0.573-0.794; $p=0.003$ ), cutoff SUVmax was 5.8 , sensitivity was $56.4 \%$, and specificity was $73.0 \%$. In

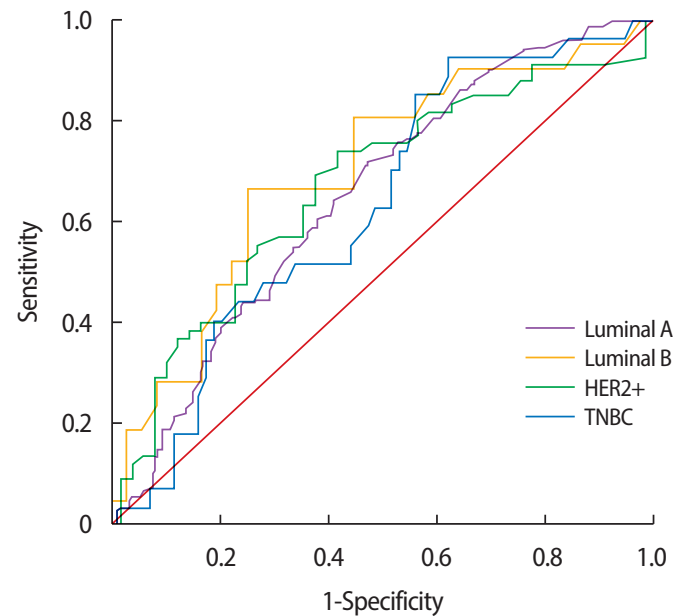

Figure 2. Receiver operating characteristic curves for the SUV max and axillary lymph node metastasis. 
Table 4. SUVmax of each molecular subtype

\begin{tabular}{lcccc}
\hline Subtype & $\begin{array}{c}\text { SUVmax } \\
(\text { mean } \pm \text { SD) }\end{array}$ & $\begin{array}{c}\text { SUVmax of Node negative patients } \\
(\text { mean } \pm \text { SD) }\end{array}$ & $\begin{array}{c}\text { SUVmax of Node positive patients } \\
\text { (mean } \pm \text { SD) }\end{array}$ & $p$-value \\
\hline Luminal $A(n=366)$ & $4.5 \pm 0.2$ & $3.9 \pm 3.2$ & $5.2 \pm 3.2$ & $<0.001$ \\
Luminal B $(n=57)$ & $6.1 \pm 0.4$ & $4.7 \pm 3.0$ & $7.0 \pm 3.8$ & 0.013 \\
HER2+ $(n=114)$ & $6.5 \pm 0.5$ & $5.3 \pm 3.9$ & $8.7 \pm 4.9$ & 0.007 \\
Triple negative $(n=95)$ & $7.5 \pm 0.5$ & $6.9 \pm 5.3$ & $8.7 \pm 4.9$ & 0.130 \\
\hline
\end{tabular}

SUVmax = maximum standardized uptake value; HER2 = human epidermal growth factor receptor 2.

Table 5. Diagnostic values and AUC of the SUV max and axillary lymph node metastasis of each subtype

\begin{tabular}{|c|c|c|c|c|c|}
\hline Variable & Overall $(n=633)$ & Luminal $A(n=366)$ & Luminal $B(n=57)$ & HER2+ $(n=114)$ & Triple negative $(n=95)$ \\
\hline Sensitivity (\%) & 70.0 & 64.7 & 56.4 & 74.2 & 63.0 \\
\hline Specificity (\%) & 54.1 & 58.8 & 73.0 & 64.6 & 51.5 \\
\hline PPV (\%) & 52.8 & 52.1 & 60.9 & 69.0 & 34.0 \\
\hline NPV (\%) & 69.8 & 70.6 & 79.4 & 55.4 & 77.8 \\
\hline AUC (95\%Cl) & $0.64(0.598-0.684)$ & $0.653(0.597-0.709)$ & $0.684(0.573-0.794)$ & $0.708(0.587-0.829)$ & $0.633(0.516-0.751)$ \\
\hline Cutoff SUVmax & 3.6 & 3.5 & 5.8 & 5.5 & 6.6 \\
\hline$p$-value & $<0.001$ & $<0.001$ & 0.003 & 0.002 & 0.043 \\
\hline
\end{tabular}

HER2 = human epidermal growth factor receptor 2; PPV= positive predictive value; NPV= negative predictive value; $A U C=$ area under curve; SUVmax = maximum standardized uptake value.

Table 6. Univariate and multivariate logistic regression analysis of axillary lymph node metastasis

\begin{tabular}{|c|c|c|c|c|}
\hline \multirow{2}{*}{ Characteristic } & \multicolumn{2}{|c|}{ Univariate } & \multicolumn{2}{|c|}{ Multivariate } \\
\hline & Odds ratio $(95 \% \mathrm{Cl})$ & $p$-value & Odds ratio $(95 \% \mathrm{Cl})$ & $p$-value \\
\hline Age & $0.94(0.68-1.29)$ & 0.691 & & \\
\hline SUVmax & $1.10(1.06-1.15)$ & $<0.001$ & $0.87(0.56-1.34)$ & 0.732 \\
\hline SUVsubmax & $2.58(1.86-3.57)$ & $<0.001$ & $2.25(1.33-3.79)$ & 0.002 \\
\hline Histologic grade (1/2 or 3 ) & $1.65(1.19-2.29)$ & 0.003 & $0.86(0.56-1.32)$ & 0.488 \\
\hline ER & $0.72(0.51-1.01)$ & 0.058 & & \\
\hline PR & $0.81(0.59-1.11)$ & 0.195 & & \\
\hline HER2 & $1.61(1.13-2.28)$ & 0.009 & $1.77(1.13-2.76)$ & 0.013 \\
\hline LVI & $8.16(5.27-12.63)$ & $<0.001$ & $7.76(4.82-12.51)$ & $<0.001$ \\
\hline Ki67 & $1.39(0.96-1.99)$ & 0.079 & & \\
\hline T stage & $4.21(3.01-5.89)$ & $<0.001$ & $2.88(1.91-4.33)$ & $<0.001$ \\
\hline
\end{tabular}

$E R=$ estrogen receptor; $P R=$ progesterone receptor; $H E R 2=$ human epidermal growth factor receptor 2; LVI= lymphovascular invasion; $S U V m a x=m a x i m u m$ standardized uptake value; SUVsubmax = subtype-adjusted maximum standardized uptake value.

the triple negative subtype, the AUC was 0.633 (95\% CI, 0.516-0.751; $p=0.043)$, cutoff SUVmax was 6.6, sensitivity was $63.0 \%$, and specificity was $51.5 \%$ (Table 5 ).

\section{Predictors of ALN metastasis}

In the univariate analysis for identifying the predictors of ALN metastasis, SUVmax, subtype-adjusted SUVmax (SUVsubmax), histological grade, HER2 positivity, LVI, and T stage were found to be significant predictors in the entire cohort. We defined the novel parameter SUVsubmax, stratifying patients above or below the subtype-optimized cut-off SUVmax (3.5 in luminal A, 5.8 in luminal B, 5.5 in
HER2+, and 6.6 in triple negative). After the multivariate analysis, SUVsubmax, HER2 positivity, LVI, and T stage remained significant predictors for ALN metastasis (Table 6). In the multivariate analysis of each subtype, we included all variables that were significant in the univariate analysis, except SUVmax and HER2 positivity because these variables do not correlate well with specific subtypes. We found that SUVsubmax was a significant predictor for the HER2+ and luminal A subtypes (Table 7). However, the association was stronger for the HER2+ subtype (odds ratio [OR], 3.5; 95\% CI, 1.23-9.76; $p=0.019$ vs. OR, 1.7; 95\% CI, 1.01-2.97; $p=0.045)$. 
Table 7. Multivariate logistic regression analysis of axillary lymph node metastasis according to molecular subtype

\begin{tabular}{|c|c|c|c|c|c|c|c|c|}
\hline \multirow[b]{2}{*}{ Variable } & \multicolumn{2}{|c|}{ Luminal A $(n=366)$} & \multicolumn{2}{|c|}{ Liminal $B(n=92)$} & \multicolumn{2}{|c|}{ HER $2+(n=80)$} & \multicolumn{2}{|c|}{ Triple negative $(n=95)$} \\
\hline & $\begin{array}{c}\text { Odds ratio } \\
(95 \% \mathrm{Cl})\end{array}$ & $p$-value & $\begin{array}{c}\text { Odds ratio } \\
(95 \% \mathrm{Cl})\end{array}$ & $p$-value & $\begin{array}{c}\text { Odds ratio } \\
(95 \% \mathrm{Cl})\end{array}$ & $p$-value & $\begin{array}{c}\text { Odds ratio } \\
(95 \% \mathrm{Cl})\end{array}$ & $p$ value \\
\hline SUVsubmax & $1.7(1.01-2.97)$ & 0.045 & $1.3(0.25-6.41)$ & 0.784 & $3.5(1.23-9.76)$ & 0.019 & $1.9(0.61-5.79)$ & 0.271 \\
\hline Histologic grade (1/2 or 3$)$ & $0.9(0.51-1.75)$ & 0.862 & $13.9(1.53-126.01)$ & 0.020 & $0.4(0.13-1.13)$ & 0.082 & $1.2(0.34-4.06)$ & 0.803 \\
\hline LVI & $8.8(4.60-16.93)$ & $<0.001$ & $7.7(0.53-110.21)$ & 0.134 & $12.2(3.44-42.98)$ & $<0.001$ & $4.7(1.49-14.93)$ & 0.009 \\
\hline T stage & $3.0(1.81-5.15)$ & $<0.001$ & $4.0(0.78-20.21)$ & 0.096 & $2.5(1.04-6.16)$ & 0.041 & $1.6(0.72-0.36)$ & 0.248 \\
\hline
\end{tabular}

HER2 = human epidermal growth factor receptor 2; LVI = lymphovascular invasion; SUVsubmax = subtype-adjusted maximum standardized uptake value.

\section{DISCUSSION}

Our findings showed that the SUVmax of primary breast cancer was positively correlated with ALN metastasis and could serve as an independent factor for metastasis prediction. Although SUVmax could predict ALN metastasis in luminal A and luminal B subtypes, the sensitivity, specificity, and robustness in the multivariate analysis were the most accurate for the HER2+ subtype.

To date, studies on the prediction of axillary metastasis using the SUVmax of different molecular subtype tumors have provided conflicting results. The study by Kim et al., in which tumors were divided into three subtypes (ER-positive/HER2 negative, HER2 positive, and triple negative), found that a high tumor SUVmax ( $\geq 4.25)$ predicted ALN metastasis in ER-positive/HER2-negative and HER2-positive subtypes, but not in the triple negative subtype [11]. In contrast, one study reported that PET was the optimal tool in detecting ALN metastasis in the triple negative subtype compared to the other three subtypes, with a sensitivity of $87.0 \%$ and specificity of $67.3 \%$ [12]. However, in this study, the ratio of the ALN to the primary tumor SUVmax was used. In a study by Song et al. [13], although they established a predictive model for ALN metastasis using the cutoff SUVmax adjusted for the molecular subtypes, subtype-specific predictive values were not included.

In our study, we found that the prediction of ALN metastasis in the triple negative subtype was not as effective as that in other subtypes. Although we could predict a statistically robust ROC curve and significant cutoff SUVmax ( $p=0.043)$ in the triple negative subtype, the comparison between patients with or without metastasis did not show a statistically significantly different SUVmax $(p=0.130)$. This may be caused by the relatively smaller number of triple negative breast cancer patients with axillary metastasis $(\mathrm{N}=27,28.4 \%)$. However, our find- ings are consistent with those of other studies indicating a lower risk of ALN metastasis in triple negative breast cancer [14]. Similar to our study, a large-scale Chinese study demonstrated that the probability of lymph node metastasis in the triple negative subtype was $28 \%$ compared with 45\% in other subtypes [15]. This observation probably reflects the distinct biology of this subtype, in which hematogenous spread is predominant over lymphatic spread [16]. Consequently, a different approach may be considered for patients with this subtype.

Despite conflicting existing results on the predictive value of SUVmax, we found that the cutoff SUVmax should be adjusted for each molecular subtype: a higher cutoff SUV is needed for more aggressive subtypes than that for less aggressive subtypes. Traditionally, a high SUVmax is related to poor prognostic indices, such as tumor grade, high proliferation index, ER negativity, and HER2 overexpression [1719]. Our results are consistent with those of previous studies, showing that, in more aggressive subtypes, such as HER2+ or triple negative, a higher SUVmax is observed [20-22]. Moreover, we found that SUVsubmax (SUVmax adjusted for molecular subtypes) was a more powerful predictor for ALN metastasis than SUVmax alone. This result suggests the necessity of establishing different cutoff SUVmax values for each molecular subtype. In one study addressing this issue, cutoff SUVmax values of 3.3, 5.5, 12.7, and 11.5 were reported for luminal A, luminal B, HER2+, and triple negative subtypes, respectively [13].

Among the predictors of ALN metastasis, LVI had the greatest OR of 7.76 (95\% CI, 4.82-12.51), followed by tumor size, with an OR of 2.88 (95\% CI, 1.91-4.33), and SUVsubmax, with an OR of 2.25 (95\% CI, 1.33-3.79). Considering that LVI and tumor size could only be accurately measured after surgery, SUVsubmax could be a useful supplementary parameter to improve the diagnostic value of PET/CT for axillary node status preoperatively.

We did not analyze the SUVmax of the ALNs. Some investigators 
have reported that the SUVmax of ALN (SUVmax LN) is also related to the avidity of the primary tumor, and the combined measure of SUVmax LN and primary tumor's SUVmax may be superior to SUV$\max$ alone [13,23-25]. However, in this study, we tried to focus on the direct association between the tumor SUVmax and ALN status because there is no reliable threshold for SUVmax LN to discriminate benign from malignant lymph nodes presently [26]. In our study, the sensitivity and specificity for the detection of ALN metastasis in the HER2+ subtype were $74.2 \%$ and $64.6 \%$, respectively. The sensitivity of PET/CT in predicting ALN metastasis ranges from 33\% to 94\% in previous studies, while the specificity is relatively higher, ranging from $66 \%$ to $100 \%$ [27]. As such, the role of PET/CT in assessing the axillary status is controversial in the field. However, as PET/CT may reflect the tumor biology, integration of the biologic information provided by PET/CT to conventional diagnostic tools may potentially expand the use of PET/CT [28].

A limitation of our study is that it was a single-center retrospective study, which might have resulted in selection bias. Moreover, as there are no standards for optimal SUVmax measurement, our results must be interpreted with care, as SUV measurement protocols may differ between institutions and may be affected by multiple factors, including blood glucose level, body composition, and partial volume effect [25]. Thus, further prospective studies with a larger cohort are required to validate our results.

In conclusion, the present study revealed that the SUVmax of primary breast cancer may be an independent predictor of ALN metastasis, most accurately in the HER2+ subtype. Our results support the preoperative use of PET/CT to facilitate a tailored axillary management for initial staging determination and treatment strategy planning in patients with breast cancer.

\section{CONFLICT OF INTEREST}

The authors declare that they have no competing interests.

\section{REFERENCES}

1. Jatoi I, Benson JR. Surgical management of the axilla in early breast cancer. Curr Probl Surg 2018;55:47-65.

2. Donker M, van Tienhoven G, Straver ME, Meijnen P, van de Velde
CJ, Mansel RE, et al. Radiotherapy or surgery of the axilla after a positive sentinel node in breast cancer (EORTC 10981-22023 AMAROS): a randomised, multicentre, open-label, phase 3 non-inferiority trial. Lancet Oncol 2014;15:1303-10.

3. Giuliano AE, Hunt KK, Ballman KV, Beitsch PD, Whitworth PW, Blumencranz PW, et al. Axillary dissection vs no axillary dissection in women with invasive breast cancer and sentinel node metastasis: a randomized clinical trial. JAMA 2011;305:569-75.

4. Lavayssiere R, Cabee AE, Filmont JE. Positron emission tomography (PET) and breast cancer in clinical practice. Eur J Radiol 2009; 69:50-8.

5. Singh D, Miles K. Multiparametric PET/CT in oncology. Cancer Imaging 2012;12:336-44.

6. Fletcher JW, Djulbegovic B, Soares HP, Siegel BA, Lowe VJ, Lyman $\mathrm{GH}$, et al. Recommendations on the use of 18F-FDG PET in oncology. J Nucl Med 2008;49:480-508

7. Veronesi U, De Cicco C, Galimberti VE, Fernandez JR, Rotmensz N, Viale G, et al. A comparative study on the value of FDG-PET and sentinel node biopsy to identify occult axillary metastases. Ann Oncol 2006;18:473-8.

8. Heusner TA, Kuemmel S, Hahn S, Koeninger A, Otterbach F, Hamami ME, et al. Diagnostic value of full-dose FDG PET/CT for axillary lymph node staging in breast cancer patients. Eur J Nucl Med Mol Imaging 2009;36:1543-50.

9. Edge SB, Compton CC. The American Joint Committee on Cancer: the 7th edition of the AJCC cancer staging manual and the future of TNM. Ann Surg Oncol 2010;17:1471-4.

10. Goldhirsch A, Wood WC, Coates AS, Gelber RT, Thurlimann B, Senn HJ. Strategies for subtypes-dealing with the diversity of breast cancer: highlights of the St. Gallen interantional expert consensus on the primary therapy of early breast cancer 211. Ann Oncol 2011; 22:1736-47

11. Kim JY, Lee SH, Kim S, Kang T, Bae YT. Tumour 18 F-FDG uptake on preoperative PET/CT may predict axillary lymph node metastasis in ER-positive/HER2-negative and HER2-positive breast cancer subtypes. Eur Radiol 2015;25:1172-81.

12. Youm JH, Chung Y, Yang YJ, Han SA, Song JY. Use of positron emission tomography-computed tomography to predict axillary metastasis in patients with triple-negative breast cancer. Korean J Clin Oncol 2018;14:135-41. 
13. Song BI, Kim HW, Won KS. Predictive Value of (18)F-FDG PET/

CT for Axillary lymph node metastasis in invasive ductal breast cancer. Ann Surg Oncol 2017;24:2174-81.

14. Mattes MD, Bhatia JK, Metzger D, Ashamalla H, Katsoulakis E. Breast cancer subtype as a predictor of lymph node metastasis according to the SEER registry. J Breast Cancer 2015;18:143-8.

15. Zhu X, Ying J, Wang F, Wang J, Yang H. Estrogen receptor, progesterone receptor, and human epidermal growth factor receptor 2 status in invasive breast cancer: a 3,198 cases study at National Cancer Center, China. Breast Cancer Res Treat 2014;147:551-5.

16. Holm-Rasmussen EV, Jensen MB, Balslev E, Kroman N, Tvedskov TF. Reduced risk of axillary lymphatic spread in triple-negative breast cancer. Breast Cancer Res Treat 2015;149:229-36.

17. De Cicco C, Gilardi L, Botteri E, Fracassi SL, Di Dia GA, Botta F, et al. Is [18F] fluorodeoxyglucose uptake by the primary tumor a prognostic factor in breast cancer? Breast 2013;22:39-43.

18. Basu S, Chen W, Tchou J, Mavi A, Cermik T, Czerniecki B, et al. Comparison of triple-negative and estrogen receptor-positive/progesterone receptor-positive/HER2-negative breast carcinoma using quantitative fluorine-18 fluorodeoxyglucose/positron emission tomography imaging parameters: a potentially useful method for disease characterization. Cancer 2008;112:995-1000.

19. Jung NY, Kim SH, Kang BJ, Park SY, Chung MH. The value of primary tumor (18)F-FDG uptake on preoperative PET/CT for predicting intratumoral lymphatic invasion and axillary nodal metastasis. Breast Cancer 2016;23:712-7.

20. Ueda S, Tsuda H, Asakawa H, Shigekawa T, Fukatsu K, Kondo N, et al. Clinicopathological and prognostic relevance of uptake level using 18F-fluorodeoxyglucose positron emission tomography/computed tomography fusion imaging (18F-FDG PET/CT) in primary breast cancer. Jpn J Clin Oncol 2008;38:250-8.

21. Specht JM, Kurland BF, Montgomery SK, Dunnwald LK, Doot RK,
Gralow JR, et al. Tumor metabolism and blood flow as assessed by positron emission tomography varies by tumor subtype in locally advanced breast cancer. Clin Cancer Res 2010;16:2803-10.

22. Koo HR, Park JS, Kang KW, Cho N, Chang JM, Bae MS, et al. 18F-FDG uptake in breast cancer correlates with immunohistochemically defined subtypes. Eur Radiol 2014;24:610-8.

23. Lovrics PJ, Chen V, Coates G, Cornacchi SD, Goldsmith CH, Law C, et al. A prospective evaluation of positron emission tomography scanning, sentinel lymph node biopsy, and standard axillary dissection for axillary staging in patients with early stage breast cancer. Ann Surg Oncol 2004;11:846-53.

24. Ahn JH, Son EJ, Kim JA, Youk JH, Kim EK, Kwak JY, et al. The role of ultrasonography and FDG-PET in axillary lymph node staging of breast cancer. Acta Radiol 2010;51:859-65.

25. Sun WY, Choi YJ, Song YJ. Prediction of axillary nodal status according to the axillary lymph node to primary breast tumor maximum standardized uptake value ratio on 18F-fluorodeoxyglucose positron emission tomography/computed tomography. J Breast Dis 2016;4:92-9.

26. Carkaci S, Adrada BE, Rohren E, Wei W, Quraishi MA, Mawlawi O, et al. Semiquantitative analysis of maximum standardized uptake values of regional lymph nodes in inflammatory breast cancer: is there a reliable threshold for differentiating benign from malignant? Acad Radiol 2012;19:535-41.

27. Crippa F, Gerali A, Alessi A, Agresti R, Bombardieri E. FDG-PET for axillary lymph node staging in primary breast cancer. Eur J Nucl Med Mol Imaging 2004;31 Suppl 1:S97-102.

28. Ahn SG, Park JT, Lee HM, Lee HW, Jeon TJ, Han K, et al. Standardized uptake value of (1)(8)F-fluorodeoxyglucose positron emission tomography for prediction of tumor recurrence in breast cancer beyond tumor burden. Breast Cancer Res 2014;16:502. 